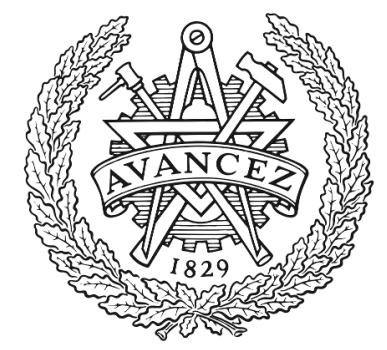

CHALMERS

UNIVERSITY OF TECHNOLOGY

\title{
Enhanced nonlinear optical susceptibilities in phosphorene nanoribbons: Ab initio study
}

Downloaded from: https://research.chalmers.se, 2023-04-26 14:00 UTC

Citation for the original published paper (version of record):

Shekarforoush, S., Shiri, D., Khoeini, F. (2018). Enhanced nonlinear optical susceptibilities in phosphorene nanoribbons: Ab initio study. Journal of Applied Physics, 123(24).

http://dx.doi.org/10.1063/1.5029547

N.B. When citing this work, cite the original published paper. 


\title{
Enhanced nonlinear optical susceptibilities in phosphorene nanoribbons: $A b$ initio study
}

\author{
Sima Shekarforoush, ${ }^{1}$ Daryoush Shiri, ${ }^{2}$ and Farhad Khoeini ${ }^{1}$ \\ ${ }^{1}$ Department of Physics, University of Zanjan, P. O. Box 45195-313, Zanjan, Iran \\ ${ }^{2}$ Department of Physics, Chalmers University of Technology, SE-41296 Göteborg, Sweden
}

(Received 15 March 2018; accepted 7 June 2018; published online 28 June 2018)

\begin{abstract}
Using density functional theory method, the linear optical absorption spectra and nonlinear optical susceptibilities of hydrogen passivated armchair and zigzag Phosphorous Nanoribbons (aPNR and zPNR) as well as $\alpha$-phase phosphorous monolayer were calculated. It was observed that the crystallographic direction has a strong effect on the band edge absorption which in turn leads to optical anisotropy as well as a red shift of the absorption spectra by increasing the width. The calculated absorption values are in the order of $10^{5} \mathrm{~cm}^{-1}$ and are very close to the experimentally measured ones. It was also observed that the 2 nd order nonlinear optical susceptibility, $\chi^{(2)}$, in the nanoribbons is enhanced by two orders of magnitude. This effect is caused by breaking the centrosymmetric structure of monolayer phosphorene as a result of hydrogen surface termination. The calculated 3rd order susceptibilities, $\chi^{(3)}$, are in the order of $\approx 10^{-13} \mathrm{esu}\left(\approx 10^{-21} \mathrm{~m}^{2} / \mathrm{V}^{2}\right)$ which are in close agreement with experimentally reported values as well as those computed based on the relativistic picture of electron. The closeness of our results to experimental values strongly supports the reliability of our method in calculating the nonlinear optical susceptibilities of phosphorene and other nanostructures in general. The enhanced 2 nd order optical nonlinearity in phosphorene promises a better second harmonic and frequency difference (THz) generation. Published by AIP Publishing. https://doi.org/10.1063/1.5029547
\end{abstract}

\section{INTRODUCTION}

Theoretical and experimental research on black phosphorous (BP) or phosphorene gained significant momentum during recent years as a result of their interesting electronic, optical, mechanical and thermal properties. Unlike graphene which lacks an energy bandgap, ${ }^{1}$ BP has a direct bandgap which is controllable by the number of layers. The medium value of bandgap $(0.6 \mathrm{eV}-2 \mathrm{eV})^{2}$ puts BP between graphene and transition metal dichalcogenides (TMD) ${ }^{3}$ and makes it a suitable choice for a high $\mathrm{I}_{\text {on }} / \mathrm{I}_{\text {off }}$ ratio field effect transistor (FET). Although the on/off ratios reported so far are in the order of $10^{4}$ which are less than that of TMD's $\left(10^{6}\right)$, they can still provide acceptable performance for analog applications, e.g., biosensors. ${ }^{4,5}$ The anisotropic band structure of phosphorene around the Brillouin Zone (BZ) center results in two different effective masses, i.e., different conductance values for electrons moving along zigzag (ZZ) or armchair (AC) directions. ${ }^{6}$ Additionally, the anisotropic phonon band structure of phosphorene monolayers leads to anisotropic mechanical properties ${ }^{7}$ and phonon-phonon scattering rates. Therefore, the thermal conductivity in this two dimensional material is anisotropic ${ }^{6}$ and its value is much less than the one for graphene which is about $3000 \mathrm{~W} / \mathrm{mK}^{8}{ }^{8}$ Vital for applications in optoelectronic devices, BP shows a direct and controllable bandgap covering a wide spectrum of photon energies (UV-IR) as well as polarization-dependent absorption, i.e., optical anisotropy. ${ }^{9}$ This means that a BP layer is transparent to light polarized along the zigzag direction due to symmetry-forbidden optical transitions; on the other hand, it is absorptive for the photons polarized along the armchair direction.
Motivated by this, many experimental studies were commenced on the linear and nonlinear optical properties of phosphorene in order to bring this material into the realm of applications, e.g., all-optical switches, limiters, phase modulators, filters, beam-splitters and polarizers among others-to name a few more examples, optical reflectance measurements, ${ }^{10}$ ultraviolet photoemission spectroscopy (UPS) and X-ray photoemission spectroscopy (XPS), angle-resolved UPS, infrared absorption due to phonons, i.e., Raman spectroscopy ${ }^{11}$ and measuring phonon dispersion by inelastic neutron scattering ${ }^{12}$ worth mentioning. These studies reveal interesting optical properties of BP including dependence of optical absorption intensity on the number of layers, doping, and polarization of photons as well as the applied normal electric field. ${ }^{13}$ It was observed that application of gate voltage leads to interesting effects like quantum-confined Franz-Keldysh effect and Pauli-blockade Burstein-Moss shift, both of which make $\alpha-P$ BP a useful infrared electro-optic material ${ }^{14}$ in sensing, spectroscopic molecular analysis, and free-space optical communications. ${ }^{15,16}$ Higher UV absorption in $\alpha$-P BP as opposed to graphene makes it more attractive for thin film solar cells. ${ }^{17}$

Experimental studies of nonlinear optics of phosphorene have shown interesting physics of carrier dynamics and electron-photon interaction mechanisms. Although the 2nd order nonlinear optical susceptibility in bulk phosphorus is small due to its centro-symmetry, ${ }^{18}$ recent studies of oddlayered exfoliated TMDs have estimated a strong second harmonic generation (SHG) in these materials. ${ }^{19}$ This observation as well as the size dependency of the 2 nd order susceptibility suggest that breaking the centro-symmetry through surface termination and/or size reduction (e.g., making nanoribbons 
or pellets) lead to the enhanced 2nd order nonlinear optical susceptibility, $\chi^{(2)}$. Enhancement of $\chi^{(2)}$ via symmetry breaking was also observed experimentally ${ }^{20-22}$ and theoretically ${ }^{23}$ for silicon nanowire-based waveguides. Furthermore, it was experimentally observed that a large nonlinearity near the band gap decreases the scattering rates and thermal losses in ultrafast optical modulation. ${ }^{24}$ Moreover, the nonlinear optical processes which occur in phosphorene (e.g., saturation of absorption (SA), reverse saturation of absorption (RSA), and two photon absorption (TPA)) are all strongly dependent on the input light intensity. ${ }^{25}$

Recent experimental studies of the 3rd order optical nonlinearity suggest that phosphorene ${ }^{26}$ and TMDs $^{27}$ have higher 3 rd order susceptibility, $\chi^{(3)}$, in comparison with graphene at the same wavelength. ${ }^{28}$ The measured experimental values $^{25,27}$ of the 3 rd order susceptibility for monolayer phosphorene are in the order of $\sim 10^{-14}$ esu. The nonlinear optical properties of black phosphorus quantum dots (BPQDs) were also investigated using $\mathrm{Z}$-scan technique, ${ }^{29}$ which shows that the 3rd order nonlinearity could be enhanced further by embedding the phosphorene dots in a liquid.

Reliable prediction of nonlinear optical susceptibilities proves useful in helping the experimenters having an estimate of the expected measurement values and pinpointing the sources of errors. Additionally, new methods of enhancing the optical nonlinearity can be envisaged by computational methods which may further lead to proposed applications for two dimensional materials.

In this work, we used time independent density functional theory implemented in SIESTA ${ }^{30}$ to calculate linear optical absorption and nonlinear optical susceptibilities ${ }^{31}$ of $\alpha$-phase monolayer phosphorene and phosphorene nanoribbons of zigzag and armchair chirality.

We observed that: First, there is a strong anisotropy in the absorption spectra of the phosphorene nanoribbons which depends on the chirality and polarization of photons. The calculated values are satisfactorily close to the experimental values of $(0.9-1) \times 10^{5} \mathrm{~cm}^{-1}$. Second, breaking the centrosymmetry of the nanoribbons due to residual stress as well as hydrogen surface termination results in a 100 fold increase of $\chi^{(2)}$ in nanoribbons.

Third, and importantly, values of $\chi^{(3)}$ for phosphorene nanoribbons and monolayer phosphorene are in close agreement with the experimentally measured values as well as an analytically calculated (perturbation-based) value of $10^{-21}$ $\mathrm{m}^{2} / \mathrm{V}^{2}{ }^{32}$ The satisfactory agreement of our numerical results with the experimental measurements validates our adopted method which is less computationally demanding than time dependent density functional theory (TDDFT) and perturbative methods.

The remaining parts of this article are organized as follows. Section II sketches all details of energy minimization, band structure calculation as well as optical calculations using DFT method implemented in SIESTA. In Sec. III, the linear absorption spectra as well as nonlinear optical susceptibilities of phosphorene nanoribbons and monolayer phosphorene are presented and discussed. A conclusion and speculation on potential applications of phosphorene is given in Sec. IV.

\section{COMPUTATIONAL METHODS}

\section{A. Energy minimization}

The energy minimization, calculation of band structure, absorption spectra and optical dipole polarization were performed using linear scale time independent density functional theory (TIDFT) method implemented in SIESTA. ${ }^{30}$ The double- $\zeta$ polarized (DZP) basis set was chosen for expansion of the electron wave function. The exchangecorrelation functional is of generalized gradient approximation (GGA) type with Perdew-Burke-Ernzerhof (PBE) pseudopotentials. ${ }^{33}$ This avoids the bandgap underestimation which is inherently caused by local density approximation (LDA) functional. The cutoff energy is $360 \mathrm{Ry}$ and the Brillouin Zone (BZ) of nanoribbons and 2D monolayer sheet were sampled with $1 \times 1 \times 9$ and $16 \times 2 \times 16$ MonkhorstPack grids, respectively. The coordinate (structural) optimization was made by conjugate gradient (CG) algorithm. The Hellmann-Feynman atomic force tolerance is equal or less than $0.015 \mathrm{eV} / \AA$. The separation between unit cells is more than $20 \AA$ in order to avoid the atomic interactions between periodic replicas of the adjacent unit cells.

\section{B. Electronic structure}

A monolayer phosphorene has a puckered honeycomb lattice in which the phosphorous (P) atoms form a $s p^{3}$ hybridization with their three nearest neighbors [See Fig. 1(a)]. Each primitive cell contains four atoms. The length of the bonds and the angles between them which were obtained after energy minimization are $\mathrm{d}_{1}=2.26 \AA, \mathrm{d}_{2}=2.28 \AA$, $\alpha=95.54^{\circ}$ and $\beta=102.35^{\circ}$, respectively. The unit cell length vectors $(\mathrm{a}=4.4923 \AA$ and $\mathrm{b}=3.3474 \AA)$ and the value of the direct bandgap energy $\left(\mathrm{E}_{\mathrm{g}}=0.95 \mathrm{eV}\right)$ were calculated by DFT. To put these obtained values in perspective with other DFT-based calculations, it is instructive to look at Table I. Figure 1(b) shows the atomic structure of unit cells for armchair and zigzag Phosphorous Nanoribbons (aPNR) and (zPNR) unit cells. Three different values for widths were chosen in the calculation of optical absorption. The name of each unit cell is the number of dimer $\mathrm{P}$ atoms which exist on the top view of each unit cell.

The corresponding width, length and bandgap values of each nanoribbon are summarized in Table II and they are in good agreement with what is reported in Ref. 34. As the width of nanoribbon is increased, the value of bandgap energy reduces and approaches to that of a monolayer which is $\approx 1 \mathrm{eV}$ (Table II). This is expected according to quantum confinement or particle in a box model. However, the trend of bandgap decrease is different for zigzag and armchair. ${ }^{35}$ It was shown that zigzag nanoribbon has a slow decaying bandgap as opposed to armchair type. The number of diatomic bonds is even and odd for zPNR and aPNR unit cells in order to facilitate the hydrogen termination along the edge of the ribbons.

Figure 2 shows the band structure and density of states of the monolayer, a $12.74 \AA$ wide zigzag nanoribbon (6-zPNR) and a $10.04 \AA$ wide armchair nanoribbon (7-aPNR), respectively. It can be observed that the 
(a)

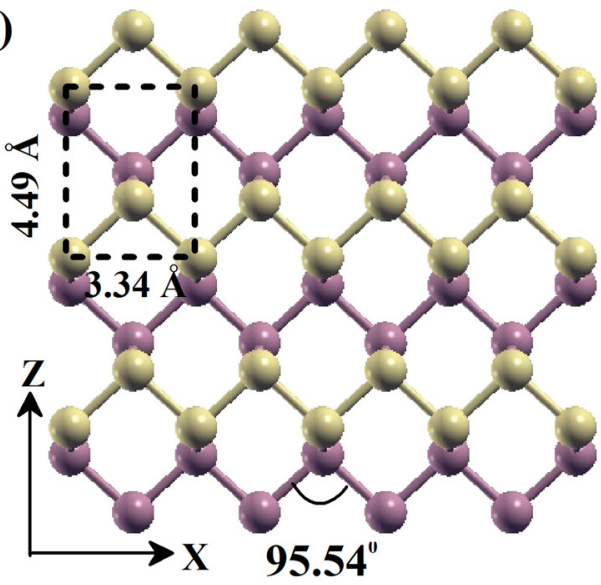

(b)

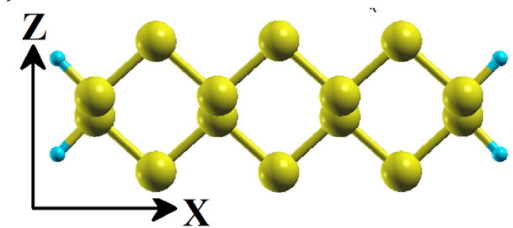

(c)

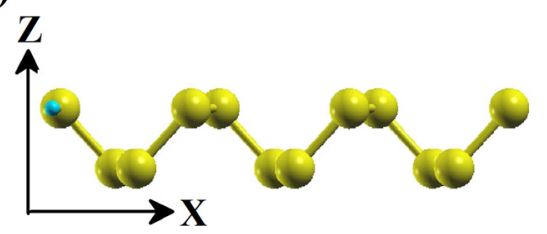

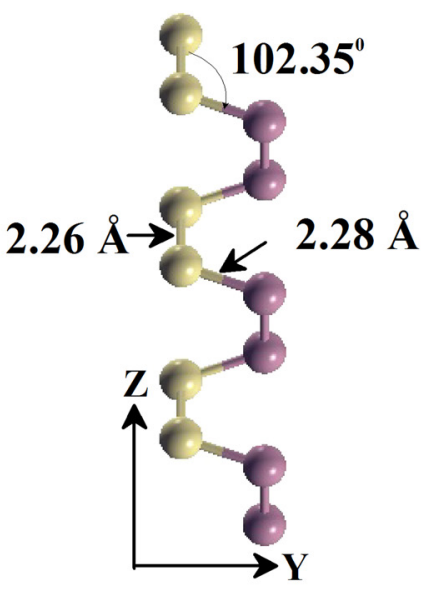
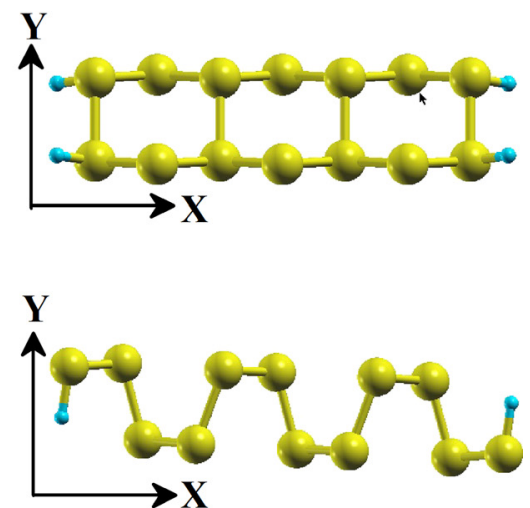

FIG. 1. (a) Left and right panels depict a section of a $2 \mathrm{D}$ monolayer phosphorene. The rectangle encloses a unit cell. The values of bond lengths, angles and unit cell lattice parameters $\left(\mathrm{d}_{1}=2.26 \AA \mathrm{d}_{2}=2.28 \AA\right.$, $\alpha=95.54^{\circ}$ and $\beta=102.35^{\circ}$ ) were obtained by SIESTA. The monolayer lies in $X Z$ plane. (b) The unit cell of an armchair nanoribbon. The ribbon is periodic along $Z$ axis and it is confined along $X$ direction. (c) The unit cell of a zigzag nanoribbon. Left/Right panels show Top/Side views of each unit cell. Yellow and blue colored atoms are phosphorous and hydrogen, respectively. monolayer phosphorene has a direct bandgap, i.e., both maximum and minimum of valence and conduction bands are at the same value of quasimomentum or in this case at the Brillouin Zone (BZ) center. The nonzero bandgap value $(0.95 \mathrm{~V})$ and its controllability with electric field ${ }^{41,42}$ offer the possibility of using phosphorene monolayers as electronic transistors with large $\mathrm{I}_{\mathrm{on}} / \mathrm{I}_{\text {off }}$ ratio. A highly anisotropic effective mass results from the sharp contrast of sub-band

TABLE I. Comparison of lattice parameters of monolayer phosphorene unit cell and the direct bandgap $\left(\mathrm{E}_{\mathrm{g}}\right)$ values obtained from our DFT method with those based on using other exchange functionals. TABLE I. is extracted and summarized from Ref. 36 to facilitate the comparison of our results with different groups.

\begin{tabular}{|c|c|c|c|c|}
\hline Method & $\mathrm{a}(\AA)$ & $\mathrm{b}(\AA)$ & $\mathrm{E}_{\mathrm{g}}(\mathrm{eV})$ & References \\
\hline PBE (SIESTA) & 4.4923 & 3.3474 & 0.95 & This work \\
\hline PBE & 4.627 & 3.298 & 0.92 & Peng et $_{\text {al. }}^{37}$ \\
\hline OptB88-vdW & 4.506 & 3.303 & 0.76 & Sa et $_{\text {al. }}^{38}$ \\
\hline HSE062@PBE & 4.627 & 3.298 & 1.54 & Sa et al. ${ }^{38}$ \\
\hline HSE062@optB88-vdW & 4.58 & 3.32 & 1.51 & Qiao et al. ${ }^{9}$ \\
\hline LDA_Mbj@optB88-vdW & 4.58 & 3.32 & 1.41 & Qiao et al. ${ }^{9}$ \\
\hline ModifiedHSE06@PBE & 4.62 & 3.35 & 1.0 & Liu et $a l .39$ \\
\hline $\mathrm{G}_{0} \mathrm{~W}_{0} @ \mathrm{PBE}$ & 4.627 & 3.298 & 2.08 & Sa et $_{\text {al. }}{ }^{38}$ \\
\hline $\mathrm{G}_{0} \mathrm{~W}_{0} @ \mathrm{PBE}$ & 4.52 & 3.31 & 1.94 & Liang et al..$^{40}$ \\
\hline G $\mathrm{G}_{0} \mathrm{~W}_{0} @ P B E \_v d W$ & $\ldots$ & $\ldots$ & 2.0 & Tran $e t a l .^{2}$ \\
\hline $\operatorname{BSE}\left(\mathrm{G}_{0} \mathrm{~W}_{0} / \mathrm{G}_{1} \mathrm{~W}_{1}\right)$ & $\ldots$ & $\ldots$ & $1.2 / 1.4$ & Tran et al. ${ }^{2,35}$ \\
\hline GW & $\ldots$ & $\ldots$ & 1.60 & Rudenko et al..$^{34}$ \\
\hline
\end{tabular}

curvatures around $\Gamma$ point (e.g., $\Gamma X$ and $\Gamma Y$ ). Due to the folding of off-center states of 2D phosphorene into the $\Gamma$ point in 1D BZ, the nanoribbons have direct bandgap as well as an effective mass for conduction band which depends on the chirality. For example, cutting the monolayer along $\Gamma X$ direction (in order to build a zigzag nanoribbon) folds the states into $\Gamma$ point and makes the band structure look like Fig. 2(b). The same scenario is valid for Fig. 2(c) where cutting an armchair nanoribbon from a monolayer causes cutting the bands along $\Gamma Y$ direction and folding them. This in turn leads to an effective mass of less value. Anisotropic effective mass adds flexibility in tuning the transport properties of electronic devices based on phosphorene. The vanHove singularities on the band edge are due to low curvature (large effective mass) valence and conduction sub-bands.

TABLE II. Width and length of the zigzag and armchair PNRs as well as the value of direct bandgap $\left(\mathrm{E}_{\mathrm{g}}\right)$ obtained after energy minimization in SIESTA.

\begin{tabular}{lccc}
\hline \hline System & Width $(\AA)$ & Length $(\AA)$ & $\mathrm{E}_{\mathrm{g}}(\mathrm{eV})$ \\
\hline 4-zPNR & 8.41 & 3.32 & 2.11 \\
6-zPNR & 12.74 & & 1.68 \\
8-zPNR & 17.10 & & 1.43 \\
5-aPNR & 6.71 & 4.27 & 1.31 \\
7-aPNR & 10.04 & & 1.12 \\
9-aPNR & 13.38 & & 1.03 \\
\hline
\end{tabular}



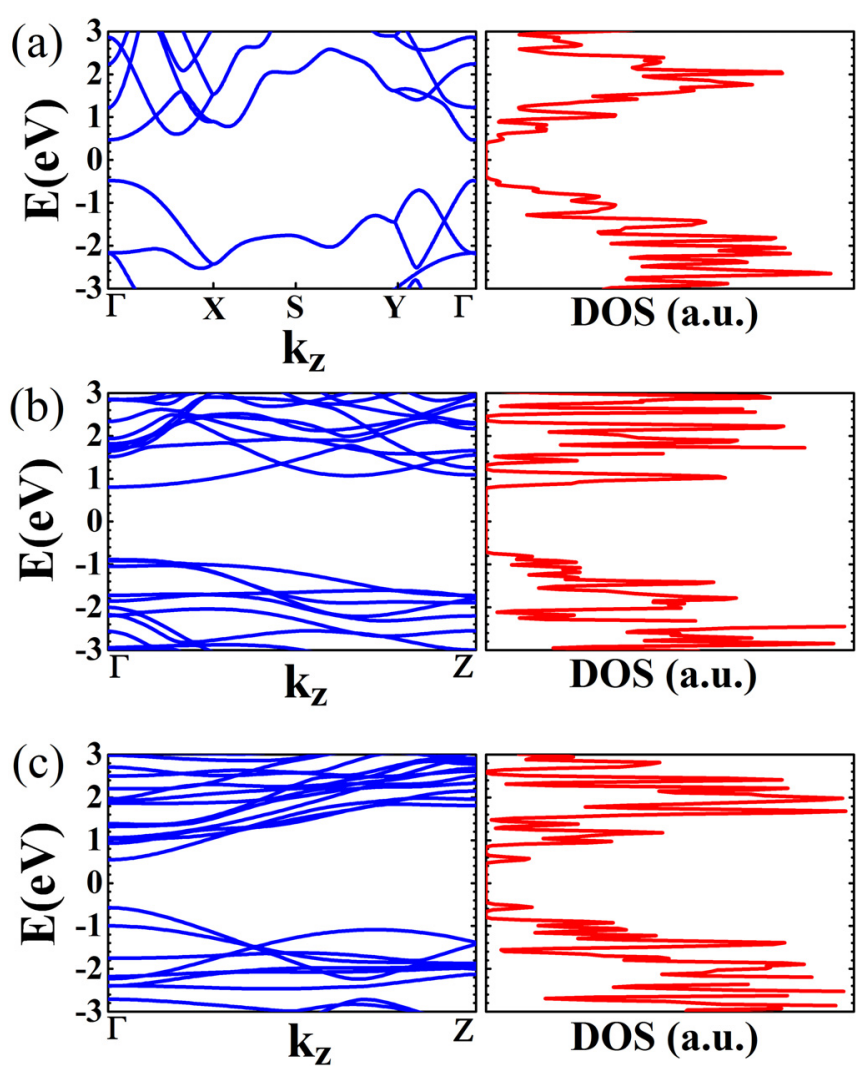

FIG. 2. Band structures and density of states for (a) monolayer phosphorene, (b) hydrogen terminated 6-zPNR and (c) 7-aPNR. The bandgap values are $0.95 \mathrm{eV}, 1.68 \mathrm{eV}$ and $1.12 \mathrm{eV}$, respectively.

\section{Linear absorption}

The optical absorption spectra of phosphorene monolayer and nanoribbons are calculated using optical functions implemented in SIESTA. ${ }^{30}$ The real part $\left(\varepsilon_{1}\right)$ and imaginary part $\left(\varepsilon_{2}\right)$ of dielectric function, $\varepsilon(\omega)$, are necessary to calculate the extinction ratio, $\kappa(\omega)$, which is

$$
\kappa(\omega)=\sqrt{\left(\sqrt{\varepsilon_{1}^{2}+\varepsilon_{2}^{2}}-\varepsilon_{1}\right) / 2},
$$

from which the absorption, $\alpha(\omega)$, is found as follows:

$$
\alpha(\omega)=\frac{4 \pi \kappa(\omega)}{\lambda_{0}}=\frac{4 \pi \kappa(\omega)}{c / f}=\frac{2 \omega \kappa(\omega)}{c},
$$

where $\lambda, c$, and $f$ are wavelength, frequency, and speed of light in vacuum, respectively. Corresponding to each electric field polarization direction $(x, y, z)$, three different spectra for absorption were obtained. The broadening energy and the value of scissor operator are $40 \mathrm{meV}$ and $1 \mathrm{eV}$, respectively.

\section{Nonlinear optical susceptibility}

Calculation of nonlinear susceptibilities in DFT starts from the calculation of polarization, $\mathbf{P}$, in response to the applied electric field, E. Since this method is based on the dipole perturbation which is induced by electric field, i.e.,

$$
H_{\text {pert }}=-\vec{E} \cdot \overrightarrow{\boldsymbol{r}}
$$

it is not applicable to periodic systems, e.g., nanoribbons and 2D monolayers. However, if the electric field is parallel with the smallest non-periodic dimensions of the system, some of the elements of susceptibility tensor can be extracted. The DFT-based methods are computationally more advantageous than time consuming perturbation-based methods. This is because in the former case, there is no need to perform integrations over many intermediate states. On the other hand, it is well known that the DFT method is reliable only at giving the correct ground state of a nano-system although this problem is circumvented by using time-dependent DFT method. ${ }^{43}$

\section{Time-dependent DFT (TDDFT)}

Although we did not use this method in our calculations, it is instructive to briefly mention its working principle. In TDDFT, the electric field is assumed to be a time dependent function, e.g., a sharp pulse. After the calculation of the ground state at $\mathrm{t}_{0}=0$, the next sample of electric field, $\boldsymbol{E}\left(t_{0}+\Delta t\right)$, is inserted in perturbation term as $H_{\text {pert }}(\Delta t)=-\vec{E}(\Delta t) \cdot \vec{r}$. Thereafter, the electron density is updated self-consistently (similar to the time-independent case of Kohn-Sham equation). The time-dependent polarization, $\boldsymbol{P}(t)$, is then found using

$$
P(t)=\int d^{3} \overrightarrow{\boldsymbol{r}} \rho(\overrightarrow{\boldsymbol{r}}, t) \overrightarrow{\boldsymbol{r}} .
$$

The polarization, $\boldsymbol{P}(t)$, includes all orders of nonlinearity in response to $E(\mathrm{t})$. Thereafter, the susceptibility values can be extracted from the Fourier transform of $\boldsymbol{P}(t)$, i.e., $\boldsymbol{P}(\omega){ }^{43}$

\section{Time-independent DFT (TIDFT)}

In this method, the polarization $(\mathbf{P})$ is calculated in response to a stepwise increase in a static electric field $(\mathbf{E})$ in SIESTA. ${ }^{30,31}$ In general, $\mathbf{P}$ has three components which depend on the electric field components along $x, y$ and $z$ directions. Generally, the tensor relation between $\mathbf{P}$ and $\mathrm{E}$ is written as ${ }^{44}$

$$
\overrightarrow{\boldsymbol{P}}=P_{0}+\epsilon_{0} \chi^{(1)} \overrightarrow{\boldsymbol{E}}+\epsilon_{0} \chi^{(2)} \overrightarrow{\boldsymbol{E}} \cdot \overrightarrow{\boldsymbol{E}}+\epsilon_{0} \chi^{(3)} \overrightarrow{\boldsymbol{E}} \cdot \overrightarrow{\boldsymbol{E}} \cdot \overrightarrow{\boldsymbol{E}}
$$

where $\mathrm{P}_{0}$ is static polarization (under zero electric field) and $\chi^{(1)}, \chi^{(2)}$ and $\chi^{(3)}$ are second, third and fourth rank tensors, respectively, i.e., they are made of $3^{2}=9,3^{3}=27$ and $3^{4}=81$ components. For a phosphorene monolayer, the electric field cannot have nonzero component along $x$ and $z$ directions. Hence, the electric field is perpendicular to the $X Z$ plane and its component in $y$ direction $\left(\mathrm{E}_{\mathrm{y}}\right)$ can be changed to calculate $\mathrm{P}_{\mathrm{x}}, \mathrm{P}_{\mathrm{y}}$ and $\mathrm{P}_{\mathrm{z}}$. As the nanoribbons are periodic along $z$ axis, only $\mathrm{E}_{\mathrm{y}}$ and $\mathrm{E}_{\mathrm{x}}$ could be nonzero. In this case, $\mathrm{P}_{\mathrm{x}}, \mathrm{P}_{\mathrm{y}}$ and $\mathrm{P}_{\mathrm{z}}$ are plotted against $E_{x}$ and $E_{y}$ and it is assumed that $E_{z}=0$. Therefore, in the first DFT step, the ground state of the energyminimized unit cell is calculated. It is further assumed that the frequency of electromagnetic field (light) and its second and third harmonics are smaller than the bandgap $\left(E_{g}\right)$, so there is no electronic excitation involved in the system. On the other hand, these frequencies should be higher than the vibrational frequencies of the nucleus. ${ }^{31}$ As an example, Eq. (5) is expanded to extract the $y$ component of polarization $\left(\mathrm{P}_{\mathrm{y}}\right)$ for a unit cell of zPNR [See Fig. 1(b)] 


$$
\begin{aligned}
P_{y}= & P_{0 y}+\epsilon_{0}\left(\chi_{y x}^{(1)} E_{x}+\chi_{y y}^{(1)} E_{y}+\chi_{y z}^{(1)} E_{z}\right)+\epsilon_{0}\left(\chi_{y x x}^{(2)} E_{x} E_{x}+\chi_{y x y}^{(2)} E_{x} E_{y}+\chi_{y x z}^{(2)} E_{x} E_{z}+\chi_{y y x}^{(2)} E_{y} E_{x}+\chi_{y y y}^{(2)} E_{y} E_{y}+\chi_{y y z}^{(2)} E_{y} E_{z}\right. \\
& \left.+\chi_{y z x}^{(2)} E_{z} E_{x}+\chi_{y z y}^{(2)} E_{z} E_{y}+\chi_{y z z}^{(2)} E_{z} E_{z}\right)+\epsilon_{0}\left\{\chi_{y x x x}^{(3)} E_{x} E_{x} E_{x}+\chi_{y x x y}^{(3)} E_{x} E_{x} E_{y}+\chi_{y x x z}^{(3)} E_{x} E_{x} E_{z}+\chi_{y x y x}^{(3)} E_{x} E_{y} E_{x}+\chi_{y x y y}^{(3)} E_{x} E_{y} E_{y}\right. \\
& +\chi_{y x y z}^{(3)} E_{x} E_{y} E_{z}+\chi_{y x z x}^{(3)} E_{x} E_{z} E_{x}+\chi_{y x z y}^{(3)} E_{x} E_{z} E_{y}+\chi_{y x z z}^{(3)} E_{x} E_{z} E_{z}+\chi_{y y x x}^{(3)} E_{y} E_{x} E_{x}+\chi_{y y x y}^{(3)} E_{y} E_{x} E_{y}+\chi_{y y x z}^{(3)} E_{y} E_{x} E_{z}+\chi_{y y y x}^{(3)} E_{y} E_{y} E_{x} \\
& +\chi_{y y y y}^{(3)} E_{y} E_{y} E_{y}+\chi_{y y y z}^{(3)} E_{y} E_{y} E_{z}+\chi_{y y z x}^{(3)} E_{y} E_{z} E_{x}+\chi_{y y z y}^{(3)} E_{y} E_{z} E_{y}+\chi_{y y z z}^{(3)} E_{y} E_{z} E_{z}+\chi_{y z x x}^{(3)} E_{z} E_{x} E_{x}+\chi_{y z x y}^{(3)} E_{z} E_{x} E_{y}+\chi_{y z x z}^{(3)} E_{z} E_{x} E_{z} \\
& \left.+\chi_{y z y x}^{(3)} E_{z} E_{y} E_{x}+\chi_{y z y y}^{(3)} E_{z} E_{y} E_{y}+\chi_{y z y z}^{(3)} E_{z} E_{y} E_{z}+\chi_{y z z x}^{(3)} E_{z} E_{z} E_{x}+\chi_{y z z y}^{(3)} E_{z} E_{z} E_{y}+\chi_{y z z z}^{(3)} E_{z} E_{z} E_{z}\right\} .
\end{aligned}
$$

Based on the above discussions, since the nanoribbon is periodic along $z$ axis, the $\mathrm{E}_{\mathrm{z}}$ should be assumed to be zero, i.e., $\mathrm{E}_{\mathrm{z}}=0 \mathrm{~V} / \AA$. As a result, $\mathrm{P}_{\mathrm{y}}$ is turned out to be a high order polynomial in terms of $E_{x}$ and $E_{y}$,

$$
\begin{aligned}
P_{y}\left(E_{x}, E_{y}\right)= & P_{0 y}+\epsilon_{0}\left(\chi_{y x}^{(1)} E_{x}+\chi_{y y}^{(1)} E_{y}\right)+\epsilon_{0}\left(\chi_{y x x}^{(2)} E_{x} E_{x}\right. \\
& \left.+\chi_{y x y}^{(2)} E_{x} E_{y}++\chi_{y y x}^{(2)} E_{y} E_{x}+\chi_{y y y}^{(2)} E_{y} E_{y}\right) \\
& +\epsilon_{0}\left\{\chi_{y x x x}^{(3)} E_{x} E_{x} E_{x}+\chi_{y x x y}^{(3)} E_{x} E_{x} E_{y}+\chi_{y x y x}^{(3)} E_{x} E_{y} E_{x}\right. \\
& +\chi_{y x y y}^{(3)} E_{x} E_{y} E_{y}+\chi_{y y x x}^{(3)} E_{y} E_{x} E_{x}+\chi_{y y x y}^{(3)} E_{y} E_{x} E_{y} \\
& \left.+\chi_{y y y x}^{(3)} E_{y} E_{y} E_{x}+\chi_{y y y y}^{(3)} E_{y} E_{y} E_{y}\right\} .
\end{aligned}
$$

As it is evident in Eq. (7), by changing $\mathrm{E}_{\mathrm{x}}$ and $\mathrm{E}_{\mathrm{y}}$ and plotting $\mathrm{P}_{\mathrm{y}}$, the susceptibility tensor components are extracted using partial derivatives of $\mathrm{P}_{\mathrm{y}}$ with respect to $\mathrm{E}_{\mathrm{x}}$ and $\mathrm{E}_{\mathrm{y}}$. For example,

$$
\chi_{y y x}^{(2)}=\frac{\partial^{2} P_{y}\left(E_{x}, E_{y}\right)}{\partial E_{y} \partial E_{x}}, \quad \chi_{y x x y}^{(3)}=\frac{\partial^{3} P_{y}\left(E_{x}, E_{y}\right)}{\partial^{2} E_{x} \partial E_{y}} .
$$

However to further simplify and speed up the calculations, the multi-dimensional surface of $\mathrm{P}_{\mathrm{y}}\left(\mathrm{E}_{\mathrm{x}}, \mathrm{E}_{\mathrm{y}}\right)$ is cut along its axis by assuming that $\mathrm{E}_{\mathrm{x}}=0$ and $\mathrm{E}_{\mathrm{y}} \neq 0$. This yields

$$
\begin{aligned}
P_{y}\left(0, E_{y}\right)= & P_{0 y}+\epsilon_{0}\left(\chi_{y y}^{(1)} E_{y}\right)+\epsilon_{0}\left(\chi_{y y y}^{(2)} E_{y} E_{y}\right) \\
& +\epsilon_{0}\left\{\chi_{y y y y}^{(3)} E_{y} E_{y} E_{y}\right\} .
\end{aligned}
$$

In the next run, it is assumed that $\mathrm{E}_{\mathrm{y}}=0$ and $\mathrm{E}_{\mathrm{x}} \neq 0$, which returns

$$
\begin{aligned}
P_{y}\left(E_{x}, 0\right)= & P_{0 y}+\epsilon_{0}\left(\chi_{y x}^{(1)} E_{x}\right)+\epsilon_{0}\left(\chi_{y x x}^{(2)} E_{x} E_{x}\right) \\
& +\epsilon_{0}\left\{\chi_{y x x x}^{(3)} E_{x} E_{x} E_{x}\right\} .
\end{aligned}
$$

The rest of the components in $\mathbf{P}$, i.e., $\mathrm{P}_{\mathrm{x}}$ and $\mathrm{P}_{\mathrm{z}}$ could also be found in a similar fashion. By fitting Eqs. (9) and (10) to the polynomials in MATLAB ${ }^{\circledR}$, the nonzero tensor components of $\chi^{(2)}$ and $\chi^{(3)}$ are extracted.

\section{RESULTS AND DISCUSSIONS}

\section{A. Linear optical absorption}

Strong optical anisotropy of monolayer phosphorene is evident after closely examining the optical absorption spectra for three different photon polarizations in Fig. 3(a).
Figures 3(b) and 3(c) show the imaginary and real parts of dielectric function, respectively. For a monolayer phosphorene subject to $x$-polarized photon, which is parallel with zigzag direction, the absorption vanishes as it is symmetry forbidden. However, it starts growing after the photon energy reaches $3.5 \mathrm{eV}$. This observation is in agreement with Ref. 9 and suggests that the valence and conduction bands along zigzag direction are of the same parity. This leads to odd symmetry for the matrix element integrand and the integral of the following type returns a zero value:

$$
\left\langle\Psi_{c}(\boldsymbol{r})|\boldsymbol{r}| \Psi_{v}(\boldsymbol{r})\right\rangle=\int \Psi_{c}^{*}(\boldsymbol{r}) \boldsymbol{r} \Psi_{v}(\boldsymbol{r}) d^{3} \boldsymbol{r} .
$$

$\Psi_{\mathrm{c}}$ and $\Psi_{\mathrm{v}}$ are conduction and valence band wave functions, respectively and $\mathbf{r}$ is the position operator. Now if the photon polarization is along $y$ direction, the absorption is negligible since the thickness of the sheet (along y direction) is extremely thin $(2.14 \AA)$. Therefore, there is an insignificant interaction between the atoms and electric field except at higher energies, e.g., above $7 \mathrm{eV}$ in Fig. 3(a). There is a strong symmetry-allowed peak on the band edge for $z$-polarized photon (red colored plot in Fig. 3). This means that armchair and zigzag direction show strong optical anisotropy which is emanating from symmetry of wave functions along these directions. When the photon polarization is rotated by $45^{\circ}$ [Fig. 3(d)], then as it is surmised, the band edge absorption drops by $50 \%$. This is because $50 \%$ of the polarization lies in parallel with the symmetry forbidden direction (zigzag) with almost zero contribution [See Inset of Fig. 3(a)].

For phosphorene nanoribbons, the quantum confinement effect is evident by examining the total absorption spectra of armchair and zigzag nanoribbons in Figs. 4(a) and 4(b), respectively. In both cases, increasing the width causes a red shift of the band edge absorption spectrum since the bandgap value decreases according to what was shown in Table II. The total absorption spectrum is the summation of three spectra corresponding to three photon polarizations. Figures 4(c) and 4(d) show the absorption spectra of $x, y$ and $z$-polarized photon for 7-aPNR and 6-zPNR, respectively. The absorption values of all structures around the band edge are agreeably close to the experimentally reported values of $(0.9$ - 1) $\times 10^{5} \mathrm{~cm}^{-1}$ for phosphorene monolayers and quantum dots. ${ }^{45}$

It is noteworthy that no absorption peak is observed for $y$-polarized cases in both nanoribbons because as it was mentioned before the thickness is small $(2.14 \AA)$ along y direction. For a $z$-polarized photon, 7-aPNR shows strong peaks 

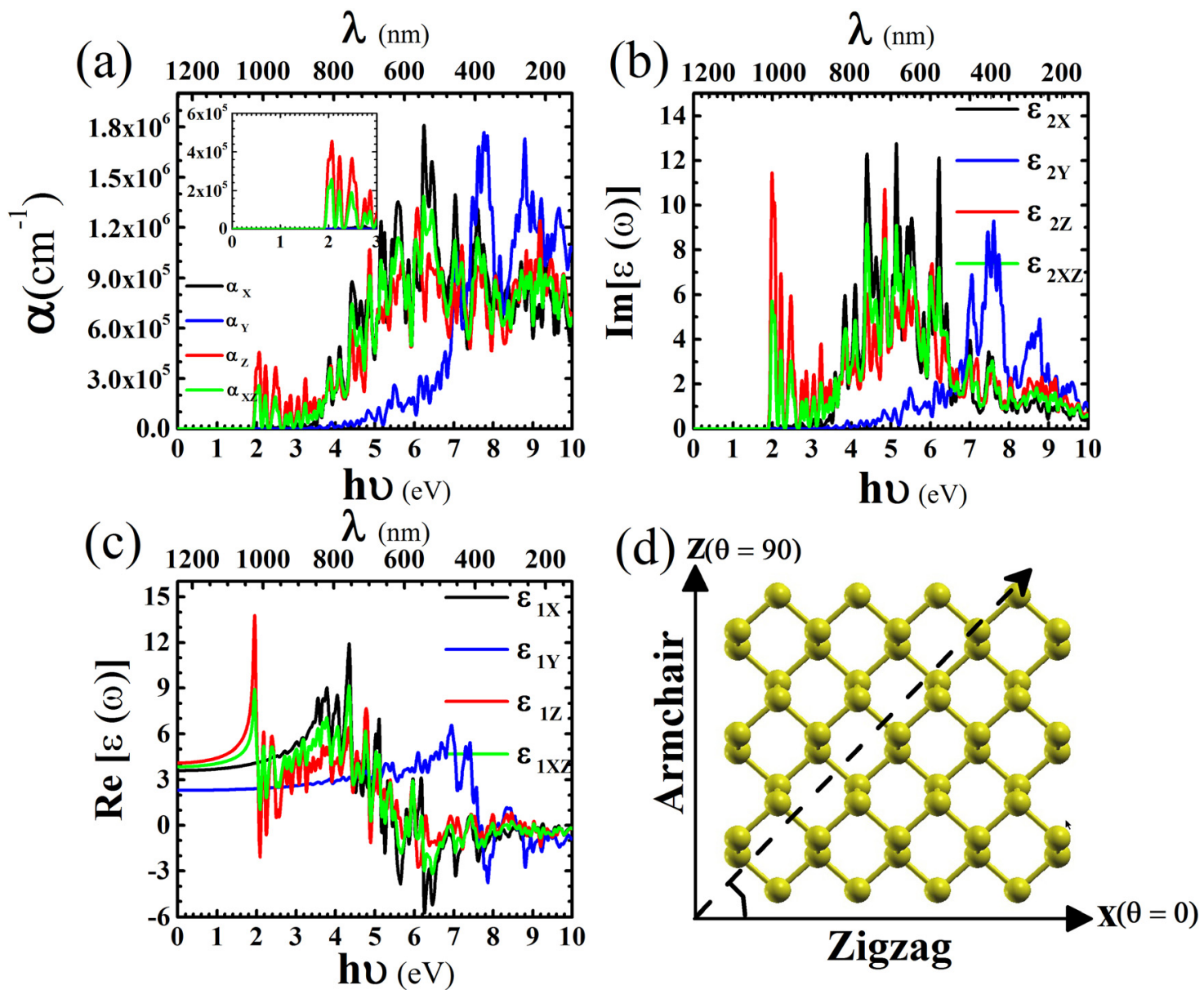

FIG. 3. (a) The optical absorption spectra, (b) imaginary part and (c) real part of the dielectric function for a monolayer phosphorene. (d) Top view of the relaxed monolayer structure. The Inset shows the band edge absorption for three different photon polarizations. The absorption is higher for z-polarized case, i.e., along the armchair edge. For a diagonally polarized (XZ) photon, the absorption drops by $50 \%$ because half of the contribution comes from $\mathrm{x}$-polarization which is zero.

as this polarization is parallel with armchair direction and the same physics as explained for optical anisotropy in monolayer phosphorene applies here. On the other hand, 6zPNR has strong absorption peaks for $x$-polarized photons as $x$ is along the armchair direction for this nanoribbon. The calculated optical anisotropy agrees well with the experimental observations of Refs. 46-48. The transparency of nanoribbon along one direction (zigzag) and the strong optical absorption along the other one (armchair) is a point of promise for applications in polarization-sensitive photodetectors. Adjustability of the band edge absorption with size could also be exploited to broaden the absorption spectrum of photodetectors and solar cells using a matrix or array of phosphorene nanoribbons with different widths.

\section{B. Nonlinear optical susceptibilities}

As mentioned before, for a phosphorene monolayer, there are two periodic directions ( $\mathrm{x}$ and $\mathrm{z}$ ), hence the electric field is only applicable along y-direction. This means that only three tensor components are extractable by fitting $\mathbf{P}(\mathrm{E})$ to polynomials. The non-zero tensor components of $\chi^{(2)}$ and $\chi^{(3)}$ are summarized in Tables III and IV, respectively. As the monolayer phosphorene is of a centro-symmetric type, ${ }^{18}$ the 2 nd order nonlinear susceptibility is very small, e.g., $\chi_{y y y}^{(2)}=0.053 \mathrm{pm} / \mathrm{V}$. In contrast to this, by confining the monolayer into nanoribbons and breaking the symmetry due to surface reconstruction, residual stress, as well as sudden surface termination with hydrogen atoms, the 2nd order susceptibility is enhanced by one and two orders of magnitude. For example, for 6-zPNR, $\chi_{y y y}^{(2)}$ and $\chi_{x y y}^{(2)}$ are $-0.184 \mathrm{pm} / \mathrm{V}$ and $-1.72 \mathrm{pm} / \mathrm{V}$, respectively. For 7 -aPNR, a tenfold enhancement is observed for $x x x$ component which is $0.125 \mathrm{pm} / \mathrm{V}$ as opposed to $0.053 \mathrm{pm} / \mathrm{V}$ for the monolayer phosphorene. This observation suggests that by breaking the centrosymmetric structure of a phosphorene monolayer, the 2nd order nonlinear effects can be enhanced up to 100 times. Converting the metric units for the 2nd and the 3rd order susceptibilities to electro-static unit (esu) is made by suitable conversion factors which are $\left(1 \mathrm{esu}=4.192 \times 10^{-4} \mathrm{~m} / \mathrm{V}\right)$ and $(1 \mathrm{esu}=1.398$ $\times 10^{-8} \mathrm{~m}^{2} / \mathrm{V}^{2}$ ), respectively. ${ }^{44}$

The calculated 3rd order susceptibilities reveal another interesting property of phosphorene nanoribbons. As it is observed in Table IV, for the monolayer phosphorene, $\chi_{\text {yyyy }}^{(3)}=3.52 \times 10^{-22} \mathrm{~m}^{2} / \mathrm{V}^{2}$ (or $2.52 \times 10^{-14} \mathrm{esu}$ ). It is noteworthy that this value is in close agreement with the experimentally observed measurements of the 3rd order susceptibility reported by Z-scan method and values extracted from two photon absorption (TPA) measurements. ${ }^{29,49}$ The experimental values 

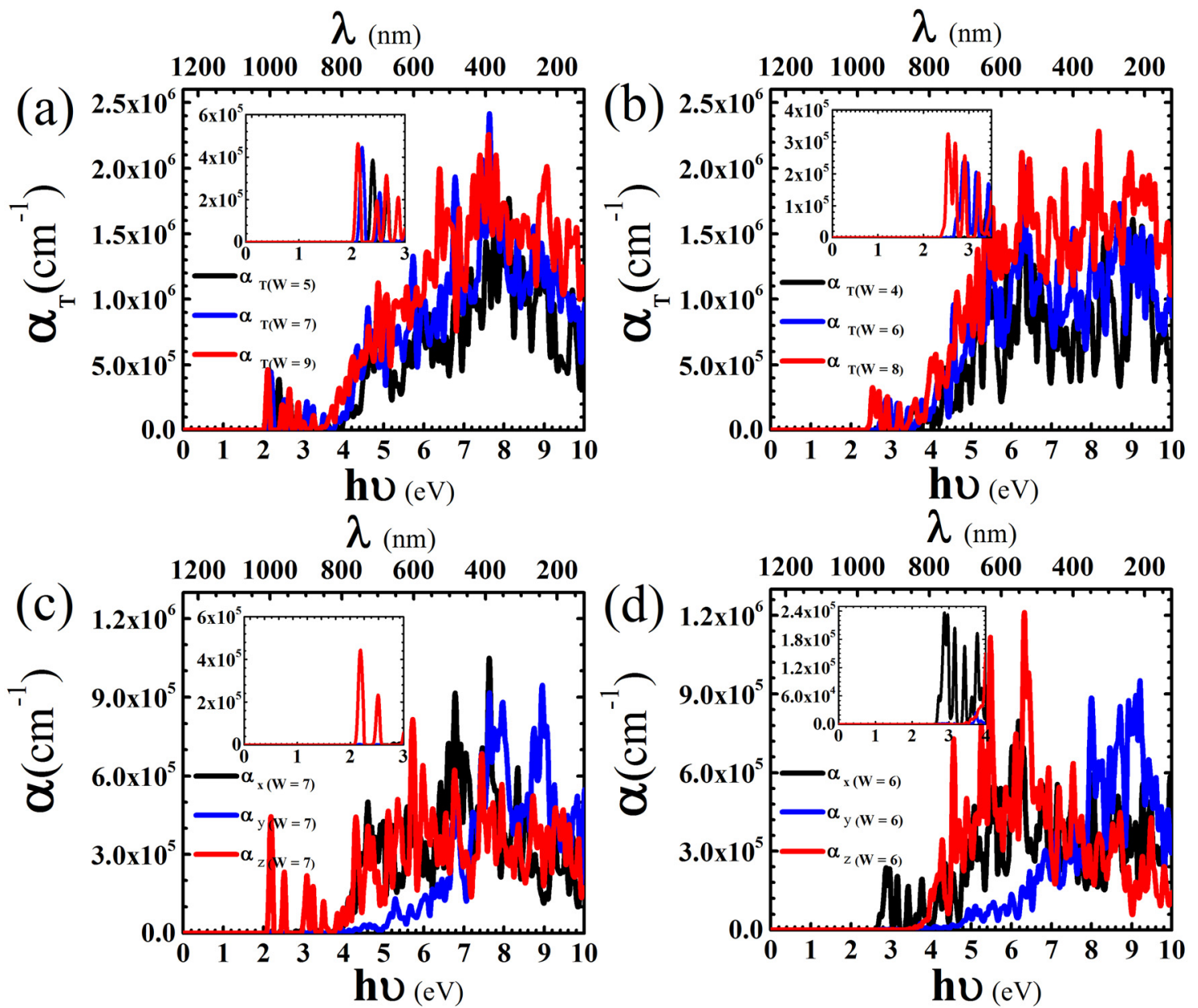

FIG. 4. Total optical absorption spectra for (a) three different widths of hydrogen-passivated aPNRs and (b) zPNRs. The absorption spectra of 7-aPNR (c) and 6-zPNR (d) for three different photon polarizations ( $\mathrm{x}, \mathrm{y} \& \mathrm{z}$ ). Black, blue and red colored plots represent $\mathrm{x}$, y and $\mathrm{z}$ polarizations, respectively.

vary from $-0.5 \times 10^{-14}$ esu to $15 \times 10^{-14} \mathrm{esu}$, depending on the wavelength used in measuring the TPA coefficient. As it is shown in Table IV, the diagonal elements of $\chi^{(3)}$ tensor, i.e., $x x x x$ and yyyy are in the order of $10^{-21} \mathrm{~m}^{2} / \mathrm{V}^{2}$. This is very close to the values calculated by analytic relativistic electron method. ${ }^{32}$ It is also noteworthy that the diagonal components of the 3rd order susceptibility (i.e., $x x x x$ and $y y y y$ ) for 6-zPNR are one order of magnitude higher than those of monolayer phosphorene. For example, $\chi_{\text {yyyy }}^{(3)}$ is $4.09 \times 10^{-21} \mathrm{~m}^{2} / \mathrm{V}^{2}$ (or $2.93 \times 10^{-13} \mathrm{esu}$ ) as opposed to $2.52 \times 10^{-14} \mathrm{esu}$ for a monolayer.

TABLE III. Non-zero 2nd order nonlinear susceptibilities of nanoribbons (6-zPNR \& 7-aPNR) and a phosphorene monolayer.

\begin{tabular}{|c|c|c|c|c|}
\hline & \multicolumn{2}{|c|}{ 6-zPNR } & \multicolumn{2}{|c|}{ 7-aPNR } \\
\hline & $\mathrm{pm} / \mathrm{V}$ & esu & $\mathrm{pm} / \mathrm{V}$ & esu \\
\hline$\chi_{x x x}^{(2)}$ & -1.72 & $-0.41 \times 10^{-8}$ & $1.25 \times 10^{-1}$ & $2.98 \times 10^{-10}$ \\
\hline$\chi_{y x x}^{(2)}$ & $8.65 \times 10^{-2}$ & $2.06 \times 10^{-10}$ & $9.74 \times 10^{-6}$ & $2.32 \times 10^{-14}$ \\
\hline$\chi_{z x x}^{(2)}$ & $5.44 \times 10^{-4}$ & $1.30 \times 10^{-12}$ & $-2.68 \times 10^{-4}$ & $-6.39 \times 10^{-13}$ \\
\hline$\chi_{y y y}^{(2)}$ & $-1.84 \times 10^{-1}$ & $-0.44 \times 10^{-9}$ & $-1.99 \times 10^{-3}$ & $-4.75 \times 10^{-12}$ \\
\hline$\chi_{x y y}^{(2)}$ & $-4.32 \times 10^{-1}$ & $-1.03 \times 10^{-9}$ & $1.10 \times 10^{-3}$ & $2.62 \times 10^{-12}$ \\
\hline$\chi_{z y y}^{(2)}$ & $-1.66 \times 10^{-3}$ & $-0.40 \times 10^{-11}$ & $8.22 \times 10^{-4}$ & $1.96 \times 10^{-12}$ \\
\hline$\chi_{y y y}^{(2)}$ & \multicolumn{4}{|c|}{$\begin{array}{c}\text { For monolayer phosphorene where both } E_{\mathrm{x}} \text { and } \mathrm{E}_{\mathrm{z}} \text { are } \\
\text { zero }-5.3 \times 10^{-2} \mathrm{pm} / \mathrm{V}=-1.27 \times 10^{-14} \mathrm{esu}\end{array}$} \\
\hline
\end{tabular}

The 3rd order nonlinear optical susceptibility for phosphorene is much smaller than the same quantity for bulk silicon and silicon nanowires, which are about $(0.1-1) \times 10^{-18}$ $\mathrm{m}^{2} / \mathrm{V}^{2}$. Experimental measurements of $\chi^{(3)}$ for graphene, $\mathrm{MoS}_{2}, \mathrm{MoSe}_{2}$ and $\mathrm{MoTe}_{2}$ using Z-scan technique ${ }^{50}$ reveal that this quantity is in the order of $10^{-14}-10^{-15}$ esu for these materials. Further enhancement of $\chi^{(3)}$ in phosphorene could be envisaged using higher number of layers, dispensing phosphorene nano pellets or (quantum dots) in a liquid solution or using a matrix of nanoribbons with mixed chirality.

TABLE IV. Non-zero 3rd order nonlinear susceptibilities of nanoribbons (6-zPNR \& 7-aPNR) and a phosphorene monolayer.

\begin{tabular}{|c|c|c|c|c|}
\hline & \multicolumn{2}{|c|}{ 6-zPNR } & \multicolumn{2}{|c|}{ 7-aPNR } \\
\hline & $\mathrm{m}^{2} / \mathrm{V}^{2}$ & esu & $\mathrm{m}^{2} / \mathrm{V}^{2}$ & esu \\
\hline$\chi_{x x x x}^{(3)}$ & $4.09 \times 10^{-21}$ & $2.93 \times 10^{-13}$ & $7.37 \times 10^{-24}$ & $5.27 \times 10^{-16}$ \\
\hline$\chi_{y x x x}^{(3)}$ & $-3.75 \times 10^{-22}$ & $-2.68 \times 10^{-14}$ & $-5.59 \times 10^{-25}$ & $-3.40 \times 10^{-17}$ \\
\hline$\chi_{z x x x}^{(3)}$ & $-2.25 \times 10^{-24}$ & $1.61 \times 10^{-16}$ & $-3.15 \times 10^{-25}$ & $-2.25 \times 10^{-17}$ \\
\hline$\chi_{y y y y}^{(3)}$ & $1.46 \times 10^{-21}$ & $1.04 \times 10^{-13}$ & $1.35 \times 10^{-22}$ & $1.89 \times 10^{-14}$ \\
\hline$\chi_{x y y y}^{(3)}$ & $-1.20 \times 10^{-23}$ & $-0.86 \times 10^{-15}$ & $3.91 \times 10^{-25}$ & $2.80 \times 10^{-17}$ \\
\hline$\chi_{z y y y}^{(3)}$ & $-6.69 \times 10^{-26}$ & $-4.79 \times 10^{-18}$ & $2.44 \times 10^{-24}$ & $1.74 \times 10^{-16}$ \\
\hline$\chi_{y y y y}^{(3)}$ & \multicolumn{4}{|c|}{$\begin{array}{c}\text { For monolayer phosphorene where both } E_{\mathrm{x}} \text { and } E_{\mathrm{z}} \text { are } \\
\text { zero } 3.53 \times 10^{-22} \mathrm{~m}^{2} / \mathrm{V}^{2}=2.52 \times 10^{-14} \mathrm{esu}\end{array}$} \\
\hline
\end{tabular}




\section{CONCLUSIONS}

We computationally investigated the linear and nonlinear optical properties of monolayer and hydrogen terminated nanoribbons of phosphorene using a time independent DFT method implemented in SIESTA. Vital for application of phosphorene in photodetectors, we observed that the band edge absorption of phosphorene is very anisotropic and depends on the incident photon polarization. Additionally, the band edge absorption shows a red shift by increasing the width in concordance with quantum confinement effect on the bandgap value and previous observations. ${ }^{35}$ The linear absorption values are in the range of $1 \times 10^{5} \mathrm{~cm}^{-1}$ which are very close to the experimentally reported values for phosphorene nano pellets and monolayers. These results suggest application of phosphorene in spectrum widening of photocells and polarization sensitive photodetectors.

By recording the electric dipole polarization $(\mathbf{P})$ in response to a static electric field (E), we extracted the 2nd and 3rd order nonlinear susceptibilities. The importance of the results is twofold. First, we computed $\chi^{(3)}$ values in close agreement with those reported in experiments as well as theoretical calculations. ${ }^{29,32,49}$ Although all components of susceptibility tensors are not extractable in our method, however, a fast and reliable approximation of these values is offered without resorting to complex and computationally demanding time dependent DFT methods.

Second, the enhanced values of $\chi^{(2)}$ in nanoribbons suggest that the centro-symmetry of monolayer phosphorene could be broken using a surface termination. This promises new applications of phosphorene nano-structures in second harmonic generation ( $\mathrm{SHG}$ ), $\mathrm{THz}$ sources, optical switches, and modulators.

\section{ACKNOWLEDGMENTS}

Daryoush Shiri acknowledges Dr. Andrei Buin at University of Toronto, Canada, for his insightful suggestions at the beginning of this project.

${ }^{1}$ K. Kim, J.-Y. Choi, T. Kim, S.-H. Cho, and H.-J. Chung, Nature 479, 338 (2011).

${ }^{2}$ V. Tran, R. Soklaski, Y. Liang, and L. Yang, Phys. Rev. B 89, 235319 (2014).

${ }^{3}$ K. F. Mak and J. Shan, Nat. Photonics 10, 216 (2016).

${ }^{4}$ Q. H. Wang, K. Kalantar-Zadeh, A. Kis, J. N. Coleman, and M. S. Strano, Nat. Nanotechnol. 7, 699 (2012).

${ }^{5}$ L. Li, Y. Yu, G. J. Ye, Q. Ge, X. Ou, H. Wu, D. Feng, X. H. Chen, and Y. Zhang, Nat. Nanotechnol. 9, 372 (2014).

${ }^{6}$ S. Lee, F. Yang, J. Suh, S. Yang, Y. Lee, G. Li, H. Sung Choe, A. Suslu, Y. Chen, C. Ko, J. Park, K. Liu, J. Li, K. Hippalgaonkar, J. J. Urban, S. Tongay, and J. Wu, Nat. Commun. 6, 8573 (2015).

${ }^{7}$ H. Chen, P. Huang, D. Guo, and G. Xie, J. Phys. Chem. C 120, 29491 (2016).

${ }^{8}$ E. Pop, V. Varshney, and A. K. Roy, MRS Bull. 37, 1273-1281 (2012).

${ }^{9}$ J. Qiao, X. Kong, Z.-X. Hu, F. Yang, and W. Ji, Nat. Commun. 5, 4475 (2014).

${ }^{10}$ T. Takahashi, K. Shirotani, S. Suzuki, and T. Sagawa, Solid State Commun. 45, 945 (1983).
${ }^{11}$ M. Ikezawa, Y. Kondo, and I. Shirotani, J. Phys. Soc. Jpn. 52, 1518 (1983).

${ }^{12}$ Y. Fujii, Y. Akahama, S. Endo, S. Narita, Y. Yamada, and G. Shirane, Solid State Commun. 44, 579 (1982).

${ }^{13}$ T. Low, A. S. Rodin, A. Carvalho, Y. Jiang, H. Wang, F. Xia, and A. H. Castro Neto, Phys. Rev. B 90, 075434 (2014).

${ }^{14}$ C. Lin, R. Grassi, T. Low, and A. S. Helmy, Nano Lett. 16, 1683 (2016).

${ }^{15}$ R. Soref, Nat. Photonics 4, 495 (2010).

${ }^{16}$ R. Stanley, Nat. Photonics 6, 409 (2012).

${ }^{17}$ J. Dai and X. Zeng, J. Phys. Chem. Lett. 5, 1289 (2014).

${ }^{18}$ J. Ribeiro-Soares, R. M. Almeida, L. G. Cancado, M. S. Dresselhaus, and A. Jorio, Phys. Rev. B 91, 205421 (2015).

${ }^{19}$ L. M. Malard, T. V. Alencar, A. P. M. Barboza, K. F. Mak, and A. M. de Paula, Phys. Rev. B 87, 201401 (2013).

${ }^{20}$ R. S. Jacobsen, K. N. Andersen, P. I. Borel, J. Fage-Pedersen, L. H. Frandsen, O. Hansen, M. Kristensen, A. V. Lavrinenko, G. Moulin, H. Ou, C. Peucheret, B. Zsigri, and A. Bjarklev, Nature 441, 199 (2006).

${ }^{21}$ M. Cazzanelli, F. Bianco, E. Borga, G. Pucker, M. Ghulinyan, E. Degoli, E. Luppi, V. Veniard, S. Ossicini, D. Modotto, S. Wabnitz, R. Pierobon, and L. Pavesi, Nat. Mater. 11, 148 (2012).

${ }^{22}$ B. Chmielak, M. Waldow, C. Matheisen, C. Ripperda, J. Bolten, T. Wahlbrink, M. Nagel, F. Merget, and H. Kurz, Opt. Express 19, 17212 (2011).

${ }^{23}$ D. Shiri, “Ab initio Study of Nonlinear Optical Susceptibilities in Silicon Nanowires," arXiv:1707.08324

${ }^{24} \mathrm{~S}$. Yu, X. Wu, Y. Wang, X. Guo, and L. Tong, Adv. Mater. 29, 1606128 (2017).

${ }^{25}$ R. Chen, Y. Tang, X. Zheng, and T. Jiang, Appl. Opt. 55, 10307 (2016).

${ }^{26}$ G. Wang, S. Zhang, X. Zhang, L. Zhang, Y. Cheng, D. Fox, H. Zhang, J. N. Coleman, W. J. Blau, and J. Wang, Photonics Res. 3, A51 (2015).

${ }^{27}$ J. Zhang, X. Yu, W. Han, B. Lv, X. Li, S. Xiao, Y. Gao, and J. He, Opt. Lett. 41, 1704 (2016).

${ }^{28}$ X. Zhang, S. Zhang, C. Chang, Y. Feng, Y. Li, N. Dong, K. Wang, L. Zhang, W. J. Blau, and J. Wang, Nanoscale 7, 2978 (2015).

${ }^{29}$ M. Liu, X.-F. Jiang, Y.-R. Yan, X.-D. Wang, A.-P. Luo, W.-C. Xu, and Z.-C. Luo, Opt. Commun. 408, 85 (2018).

${ }^{30}$ J. M. Soler, E. Artacho, J. D. Gale, A. Garca, J. Junquera, P. Ordejn, and D. Snchez-Portal, J. Phys.: Condens. Matter 14, 2745 (2002).

${ }^{31}$ N. Berkaine, E. Orhan, O. Masson, P. Thomas, and J. Junquera, Phys. Rev. B 83, 245205 (2011).

${ }^{32}$ T. G. Pedersen, Phys. Rev. B 95, 235419 (2017).

${ }^{33}$ J. P. Perdew, K. Burke, and M. Ernzerhof, Phys. Rev. Lett. 78, 1396 (1997).

${ }^{34}$ A. N. Rudenko and M. I. Katsnelson, Phys. Rev. B 89, 201408 (2014).

${ }^{35}$ V. Tran and L. Yang, Phys. Rev. B 89, 245407 (2014).

${ }^{36}$ Y. Jing, X. Zhang, and Z. Zhou, Wiley Interdiscip. Rev.: Comput. Mol. Sci. 6, 5-19 (2016).

${ }^{37}$ X. Peng, Q. Wei, and A. Copple, Phys. Rev. B 90, 085402 (2014).

${ }^{38}$ B. Sa, Y.-L. Li, J. Qi, R. Ahuja, and Z. Sun, J. Phys. Chem. C 118, 26560 (2014).

${ }^{39}$ H. Liu, A. Neal, Z. Zhu, Z. Luo, X. Xu, D. Tomanek, and P. Ye, ACS Nano 8, 4033 (2014).

${ }^{40}$ L. Liang, J. Wang, W. Lin, B. G. Sumpter, V. Meunier, and M. Pan, Nano Lett. 14, 6400 (2014).

${ }^{41}$ M. Ezawa, New J. Phys. 16, 115004 (2014).

${ }^{42}$ S. Shekarforoush, D. Shiri, and F. Khoeini, "Spin-orbit coupling effects and electromechanical response in phosphorene nanoribbons" (unpublished).

${ }^{43}$ Y. Takimoto, F. D. Vila, and J. J. Rehr, J. Chem. Phys. 127, 154114 (2007).

${ }^{44}$ R. Boyd, Nonlinear Optics, 3rd ed. (Academic Press, 2008).

${ }^{45}$ R. Chen, X. Zheng, and T. Jiang, Opt. Express 25, 7507 (2017).

${ }^{46}$ D. Li, H. Jussila, L. Karvonen, G. Ye, H. Lipsanen, X. Chen, and Z. Sun, Sci. Rep. 5, 15899 (2015).

${ }^{47}$ H.-Y. Wu, Y. Yen, and C.-H. Liu, Appl. Phys. Lett. 109, 261902 (2016).

${ }^{48}$ X. Wang and S. Lan, Adv. Opt. Photonics 8, 618 (2016).

${ }^{49} \mathrm{~S}$. Zhang et al., Optical. Material Express 6(1), 3159-3168 (2016).

${ }^{50}$ K. Wang, Y. Feng, C. Chang, J. Zhan, C. Wang, Q. Zhao, J. N. Coleman, L. Zhang, W. J. Blau, and J. Wang, Nanoscale 6, 10530 (2014). 